\title{
Spatial Reflection and Associated String Order in Quantum Spin Chains
}

\author{
Li-Xiang Cen \\ Department of Physics, Sichuan University, Chengdu 610065, China
}

\begin{abstract}
We investigate spatial reflection and associated nonlocal order in spin chain quantum systems. The proposed string order parameters, e.g., reflected via operations of the spatial reflection or combinations of it with spin reflection, are able to characterize a variety of physical systems and allow us to gain renewed insights to the statistical mechanism underlying phenomena such as the Haldane gap and quantum phase transitions. Besides revealing further the potential application of the generalized parity symmetry in numerical algorithm, we build an explicit scheme to determine the symmetry and the related string order for matrix product states so that one can construct ansatz models with presumed properties.
\end{abstract}

PACS numbers: 75.10.Pq, 03.67.-a, 64.70.Tg, 02.20.Qs

Order parameter has been one of the most important concepts in condensed matter physics since Landau's finding of it in describing continuous phase transitions with spontaneous symmetry breaking. The nonlocal string order, originally introduced by den Nijs and Rommelse $\frac{1}{2}$, was thought of a hidden antiferromagnetic Néel order and exploited to understand the mechanism of the Haldane gap 2 . Despite that Haldane ground states of integer- $S$ Heisenberg chains possess only short-range spin-spin correlations, they could have nonzero string order parameters. The latter was found to be a common feature of the Haldane phase resulted from breaking of certain topological symmetry $\underline{3}^{\underline{3}}$ and the phenomenon has been investigated for various physical models ${ }^{4}$. A typical example is the valence bond solid (VBS) model ${ }^{5}$ and its higher- $S$ generalizations $\frac{6,7}{}$, in which the string order parameter can be explicitly worked out.

To determine the hidden order for a general quantum many-body system is a problem highly nontrivial. In a recent literature $e^{-}$, the presence of den Nijs-Rommelse string order is demonstrated rigorously in relation to the existence of local symmetries within the framework of matrix product states (MPSs). The revealed connection is somewhat universal in the sense that the MPS formulation indeed offers a general mathematical representation for quantum many-body states ${ }^{9,10,11}$. As a consequence, the problem of determining the den Nijs-Rommelse string order for a quantum system is recast as that of finding out possible local symmetries in the system. At this stage, it is of interest to build a scheme to identify the local symmetry for general MPSs, since for every MPS with a finite representative dimension an ansatz model with local interactions can always be constructed ${ }^{9}$ such that the given MPS constitutes its ground state.

In this paper we investigate the spatial reflection and propose novel string order to characterize spin lattice systems. Differing from conventional string operators utilizing local unitary transformations, the present string operator acting on spins in between two boundaries is defined by the spatial reflection or combinations of it with local spin reflection. By virtue of the MPS representation, the proposed string order is shown to relate intimately to the symmetry with respect to parity or generalized parity transformations. The quantity is then applied to characterize a sort of models including the VBS states and a spin- $\frac{1}{2}$ system with quantum phase transitions. Similar to the parity symmetry, the generalized parity symmetry could also be applied to reduce computational costs in numerical algorithm of the density matrix renormalization group (DMRG) $\underline{12,13}$. In addition, we build an explicit protocol to determine from first principles the string order for MPSs with representative dimension $D=2$ and present an example of modeling ansatz with presumed properties.

The primitive form of the string order parameter employed here is proposed as

$$
\mathrm{SO}_{P}^{\alpha} \equiv \lim _{|j-i| \rightarrow \infty}\left\langle S_{i}^{\alpha} \mathcal{P}^{(l)} S_{j}^{\alpha}\right\rangle,
$$

where $S_{i}^{\alpha}(\alpha=x, y, z)$ denotes the spin operator of the $i$ th lattice site and the parity operator acts on $l=j-i-1$ consecutive sites between the boundary spins $i$ and $j$ as $\mathcal{P}^{(l)}\left|s_{i+1} \cdots s_{i+l}\right\rangle=\left|s_{i+l} \cdots s_{i+1}\right\rangle$. This string operator and its generalized form of Eq. (10) can be exploited to characterize the hidden order for infinite lattice systems just as the original den Nijs-Rommelse string order.

To be specific, let us resort to the MPS formulation for translationally invariant spin chains

$$
|\Psi\rangle=\frac{1}{\sqrt{\operatorname{Tr} E^{N}}} \sum_{\left\{s_{i}\right\}} \operatorname{Tr}\left(A^{s_{1}} \cdots A^{s_{N}}\right)\left|s_{1}, \cdots, s_{N}\right\rangle,
$$

where $s_{i}=1, \cdots, d$ specifies the lattice spin degrees of freedom and $\left\{A^{s}\right\}$ is a set of $D \times D$ matrices parameterizing the state $|\Psi\rangle$. The transfer matrix $E$ contained in the normalization factor is given as $E=$ $\sum_{s=1}^{d}\left(A^{s}\right)^{*} \otimes A^{s}$. By using further the notations, say, $E_{S^{\alpha}} \equiv \sum_{s, s^{\prime}}\left\langle s\left|S^{\alpha}\right| s^{\prime}\right\rangle\left(A^{s}\right)^{*} \otimes A^{s^{\prime}}$, the quantity $\mathrm{SO}_{P}$ of the state $|\Psi\rangle$ is shown to be

$$
\mathrm{SO}_{P}^{\alpha}=\lim _{l \rightarrow \infty} \lim _{N \rightarrow \infty} \frac{\operatorname{Tr}\left[E_{S_{i}^{\alpha}} E_{\mathcal{P}^{(l)}} E_{S_{j}^{\alpha}} E^{N-l-2}\right]}{\operatorname{Tr} E^{N}},
$$

where

$$
E_{\mathcal{P}^{(l)}}=\left[\left(E^{T_{2}}\right)^{l}\right]^{T_{2}}, \quad E^{T_{2}}=\sum_{s}\left(A^{s}\right)^{*} \otimes\left(A^{s}\right)^{T} .
$$


Note that the state $|\Psi\rangle$ is identified to be parity symmetrio $\frac{14,15}{15}$ iff there is $\left\langle\Psi\left|\mathcal{P}^{(N)}\right| \Psi\right\rangle=$ $\operatorname{Tr}\left(E^{T_{2}}\right)^{N} / \operatorname{Tr} E^{N}= \pm 1$. The latter means that for an infinite spin chain the largest eigenvalue $\lambda_{m}^{\prime}$ of $E^{T_{2}}$ should have the same modulus with the $\lambda_{m}$ of $E$. This fact together with the relation $\lim _{l \rightarrow \infty} E_{\mathcal{P}^{(l)}}=\lambda_{m}^{\prime l}\left(\left|\Phi_{m}^{R}\right\rangle\left\langle\Phi_{m}^{L}\right|\right)^{T_{2}}$ lead to that the quantity $\mathrm{SO}_{P}^{\alpha}$ could be nonzero iff the state $|\Psi\rangle$ is parity symmetric and the corresponding value is calculated as

$$
\mathrm{SO}_{P}^{\alpha}=\frac{1}{\lambda_{m}^{2}}\left\langle\Psi_{m}^{L}\left|E_{S_{i}^{\alpha}}\left(\left|\Phi_{m}^{R}\right\rangle\left\langle\Phi_{m}^{L}\right|\right)^{T_{2}} E_{S_{j}^{\alpha}}\right| \Psi_{m}^{R}\right\rangle
$$

where $\left|\Psi_{m}^{L(R)}\right\rangle$ and $\left|\Phi_{m}^{L(R)}\right\rangle$ denote respectively the left (right) eigenvectors of $E$ and $E^{T_{2}}$ related to $\lambda_{m}$.

Let us address a concrete example of the VBS ground state of the Affleck-Kennedy-Lieb-Tasaki model ${ }^{5}$ represented by $\left\{A^{s}\right\}=\left\{\sigma_{z}, \sqrt{2} \sigma_{+}, \sqrt{2} \sigma_{-}\right\}$. In this case, $E$ and $E^{T_{2}}$ are Hermitian thus their dual bases of left and right eigenvectors recover simply the conventional orthonormal bases. Their largest eigenvalue $\lambda_{m}=3$ and the corresponding eigenvectors are $\left|\Psi_{m}\right\rangle=\frac{1}{\sqrt{2}}(|00\rangle+|11\rangle)$ and $\left|\Phi_{m}\right\rangle=\frac{1}{\sqrt{2}}(|01\rangle-|10\rangle)$. The string order $\mathrm{SO}_{P}$ is worked out to be $\left|\mathrm{SO}_{P}^{\alpha}\right|=\frac{1}{18}$, which is independent of the orientation $\alpha=x, y$ or $z$ owing to the $\mathrm{SU}(2)$ symmetry of the model. Moreover, the extended model of the $\mathrm{SO}(5)$ matrix product state ${ }^{7.16}$ specified by

$$
A^{0}=\sigma_{z} \otimes \sigma_{z}, A^{ \pm 1}=\sqrt{2} \sigma_{z} \otimes \sigma_{ \pm}, A^{ \pm 2}=\sqrt{2} \sigma_{ \pm} \otimes I
$$

has also nonzero $\mathrm{SO}_{P}$. The corresponding matrices $E$ and $E^{T_{2}}$ are Hermitian again and have the largest eigenvalues $\lambda_{m}=5$ with eigenvectors as

$$
\left|\Psi_{m}\right\rangle=\frac{1}{2} \sum_{i=1}^{4}|i i\rangle,\left|\Phi_{m}\right\rangle=\frac{1}{2}(|14\rangle-|41\rangle+|32\rangle-|23\rangle) .
$$

In terms of the $S^{z}$ quantum number of the $s=2$ lattice spins, the string order is obtained as $\mathrm{SO}_{P}^{z}=-\frac{1}{5}$.

For further application let us consider a spin- $\frac{1}{2}$ matrix product system specified by $A^{0}=\left(\begin{array}{cc}0 & 0 \\ 1 & 1\end{array}\right), A^{1}=\left(\begin{array}{cc}1 & g \\ 0 & 0\end{array}\right)$. This is an ansatz mode $1^{17}$ described by a parent Hamiltonian with three-body interactions and it undergoes a quantum phase transition at the point $g=0$. The system is parity symmetric and the matrices $E$ and $E^{T_{2}}$ have the same eigenvalues $\lambda_{ \pm}=1 \pm g$. By definition, the string order parameter $\mathrm{SO}_{P}^{z}$ of the ground MPS is shown to take a discrete form with $\mathrm{SO}_{P}^{z}=\frac{1}{8}$ as $g<0$ and $\mathrm{SO}_{P}^{z}=\frac{1}{8}\left(\frac{1-g}{1+g}\right)^{2}$ as $g>0$. On the other hand, the system possesses also a local $Z_{2}$ symmetry with respect to the spin flip $|0\rangle \leftrightarrow|1\rangle$. The transverse den Nijs-Rommelse string order associated with the local unitary $U=\exp \left(i \pi S^{x}\right)$, namely,

$$
\mathrm{SO}_{D}^{x} \equiv \lim _{|j-i| \rightarrow \infty}\left\langle S_{i}^{x} U^{\otimes l} S_{j}^{x}\right\rangle, \quad l=j-i-1,
$$

is obtained as $\left|\mathrm{SO}_{D}^{x}\right|=g /(1+g)^{2}$ or $\mathrm{SO}_{D}^{x}=0$ for cases $g>0$ and $g<0$, respectively. Thus the quantum phase transition of the system crossing the point $g=0$ is marked distinctly by the non-analytical behavior of both these string order parameters.

As the quantity $\mathrm{SO}_{P}$ reveals the hidden order for states with parity symmetry, we show below that the state with generalized symmetry with respect to combination of the parity and spin reflection indicates another type of nonlocal order. For a simple example consider an MPS 15 represented by $\left\{A^{s}\right\}=\left\{\left(\begin{array}{cc}1 & 0 \\ 0 & \sqrt{2}\end{array}\right),\left(\begin{array}{ll}0 & 0 \\ \sqrt{2} & 0\end{array}\right),\left(\begin{array}{ll}0 & 1 \\ 0 & 0\end{array}\right)\right\}$. The state is parity absent but invariant under the combined operation $\mathcal{P}^{(N)} U_{P}^{\otimes N}$, where $U_{P}$ is a spin flip operator specified as $U_{P}=|1\rangle\langle 1|+| 2\rangle\langle 3|+| 3\rangle\langle 2|$. This sort of generalized parity symmetry is identified for MPSs as there is

$$
\left(A^{i}\right)^{T}=\sum_{j=1}^{d} U_{P}^{i j}\left(X A^{j} X^{-1}\right), \quad i=1, \cdots, d
$$

and it is readily verified that for the above example there exists $X=\left(\begin{array}{cc}\sqrt{2} & 0 \\ 0 & 1\end{array}\right)$. Indeed, the kind of symmetry was already known in fermion systems in performance of the DMRG procedure $\frac{13}{}$. Owing to the fermion anti-commutation rule, the Jordan-Wigner transformation will result in a parity asymmetric Hamiltonian which is, however, invariant under the combination of the parity and a simple spin reflection.

Accordingly, one is led to consider a string operator

$$
\mathrm{SO}_{G P}^{\alpha} \equiv \lim _{|j-i| \rightarrow \infty}\left\langle S_{i}^{\alpha} \mathcal{P}^{(l)} U_{P}^{\otimes l} S_{j}^{\alpha}\right\rangle,
$$

where $\mathcal{P}^{(l)} U_{P}^{\otimes l}$ describes the combined transformation on $l=j-i-1$ consecutive lattice sites in between $i$ and $j$. For an MPS with generalized parity symmetry specified by Eq. (9), this string quantity is shown to be

$$
\mathrm{SO}_{G P}^{\alpha}=\frac{\left\langle\Psi_{m}^{L}\left|E_{S_{i}^{\alpha}} V^{-1}\left(\left|\Psi_{m}^{R}\right\rangle\left\langle\Psi_{m}^{L}\right|\right)^{T_{2}} V E_{S_{j}^{\alpha}}\right| \Psi_{m}^{R}\right\rangle}{\lambda_{m}^{2}},
$$

where $V \equiv I \otimes X,\left|\Psi_{m}^{L, R}\right\rangle$ are eigenvectors of $E$ related to $\lambda_{m}$, and the relation $\lim _{l \rightarrow \infty}\left(E^{l}\right)^{T_{2}}=\lambda_{m}^{l}\left(\left|\Psi_{m}^{R}\right\rangle\left\langle\Psi_{m}^{L}\right|\right)^{T_{2}}$ has been applied to obtain the equality. The string order of the MPS mentioned above Eq. (9) is then derived as $\mathrm{SO}_{G P}^{z}=\frac{7}{81}$ and $\mathrm{SO}_{G P}^{x}=-\mathrm{SO}_{G P}^{y}=\frac{4}{81}$.

Revelation of the existence of the generalized parity symmetry in spin lattice systems is important in many aspects. For instance, it can be exploited to reduce computational costs in numerical algorithm. In fact, it has long been realized in the DMRG algorithm $\frac{12}{}$ that for systems with parity symmetry the density matrix of environment blocks could be achieved via a simple reflection on that of the system blocks. The various existence of generalized parity symmetries can also be utilized to achieve a factor $\sim 2$ speedup and the density matrices of system and environment blocks are now connected via the generalized parity transformation. Moreover, in view that an MPS can be viewed as a ground state of an ansatz system with short-range interactions, it is of interest to construct the parent Hamiltonian of the modeling system with certain presumed parity symmetry. The latter 
implies that the derived system might possess nonlocal order associated with the specified string operator.

We now focus on the problem of determining the generalized parity symmetry for MPSs so as to construct ansatz lattice models with prescribed properties. The question arises as: given an MPS $|\Psi\rangle$, how to certify the relation (9) exists or not and how to find out such $U_{P}$ and $X$ if they do exist? To this end, we employ an expression equivalent with Eq. (9) but in terms of the transfer matrix

$$
E^{T}=\left(X^{*} \otimes X\right) E\left(X^{*} \otimes X\right)^{-1} .
$$

In comparison with Eq. (9), this equation focuses solely on the invertible matrix $X$ and the task reduces to distinguish a particular $S$, among all those satisfying $E^{T}=$ $S E S^{-1}$, that could be decomposed into $S=X^{*} \otimes X$. The difficulty of the problem comes from that the similar transformation $S$ connecting $E^{T} \sim E$ is not unique and the complexity even increases with representative dimensions. Intriguingly, we show below that for cases of $D=2$ the problem could be resolved by virtue of a particular realization of group homomorphism between the direct product group of unimodular linear transformations and the four-dimensional complex orthogonal group $\frac{18}{}$.

Briefly, it was shown in Ref. 18 that in terms of a sort of so-called pseudo-orthonormal bases, the representative matrix of the operator $X_{1} \otimes X_{2}$, where $X_{1,2}$ denote arbitrary linear transformation of $\mathrm{SL}(2, \mathrm{C})$, is complex orthogonal and constitutes a group element of $\mathrm{SO}(4, \mathrm{C})$. The result leads to an intuitive perception that the pseudo-orthonormal bases could be utilized to resolve the factorization problem for the mentioned similar transformation $S$. To be specific, the following simplest pseudoorthonormal bases (the so-called "magic bases", see Ref. 19) will be employed

$$
\begin{aligned}
& \left|e_{1}\right\rangle=\frac{\sqrt{2} i}{2}(|00\rangle+|11\rangle),\left|e_{2}\right\rangle=\frac{\sqrt{2}}{2}(|00\rangle-|11\rangle), \\
& \left|e_{3}\right\rangle=\frac{\sqrt{2}}{2}(|01\rangle+|10\rangle),\left|e_{4}\right\rangle=\frac{\sqrt{2} i}{2}(|01\rangle-|10\rangle) .
\end{aligned}
$$

Suppose that the transfer matrix has full rank and is expressed as $E=\sum_{i=1}^{4} \lambda_{i}\left|\Psi_{i}^{R}\right\rangle\left\langle\Psi_{i}^{L}\right|$ according to its spectrum structure. Its transposition is then formed as $E^{T}=\sum_{i=1}^{4} \lambda_{i}\left|\Psi_{i}^{L *}\right\rangle\left\langle\Psi_{i}^{R *}\right|$ and the series of similar transformations connecting them are given by $S\left(k_{i}\right)=$ $\sum_{i=1}^{4} k_{i}\left|\Psi_{i}^{L *}\right\rangle\left\langle\Psi_{i}^{L}\right|$, where the parameters $k_{i} \neq 0$ are to be determined later. The distinguishing problem of Eq. (12) could be resolved according to the following protocol: i) Express the operator $S\left(k_{i}\right)$ in terms of bases (13) and denote the representative matrix as $D_{\mu \nu}^{s}\left(k_{i}\right)=\left\langle e_{\mu}\left|S\left(k_{i}\right)\right| e_{\nu}\right\rangle$; ii) Presume that $D^{s}\left(D^{s}\right)^{T}=$ $\left(D^{s}\right)^{T} D^{s}=I$ and resolve the set of $k_{i}$ if they do exist; iii) Factorize the operator as $S\left(k_{i}\right)=X_{1} \otimes X_{2}$ according to the correspondence of the group homomorphism $\mathrm{SO}(4, \mathrm{C}) \sim \mathrm{SL}(2, \mathrm{C}) \otimes \mathrm{SL}(2, \mathrm{C})$ and verify if there exists $X_{2}=X_{1}^{*}$. Once $X$ is figured out, the spin reflection $U_{P}$ can be worked out easily from Eq. (9).
It is worthy to note that a slightly modified scheme is capable to determine the local symmetry hence the string order $S O_{D}$ for MPSs, wherein the condition reads as ${ }^{8} A^{i}=\sum_{j} U^{i j}\left(X A^{j} X^{-1}\right)$. The problem then becomes to find out a decomposable transformation $S=X^{*} \otimes$ $X$ from all those satisfying $E=S E S^{-1}$. The $S$ here is expressed as $S\left(k_{i}\right)=\sum_{i} k_{i}\left|\Psi_{i}^{R}\right\rangle\left\langle\Psi_{i}^{L}\right|$ by invoking the spectrum structure of $E$. The distinguishing problem can be resolved following the same protocol described above.

Below we present an example to illustrate the scheme and construct the interaction model with presumed symmetry. Consider a family of MPSs $|\Psi(g)\rangle$ with

$$
A^{0}=\left[\begin{array}{ll}
1 & 0 \\
0 & g
\end{array}\right], A^{+}=\left[\begin{array}{ll}
0 & 0 \\
g & 0
\end{array}\right], A^{-}=\left[\begin{array}{ll}
0 & 1 \\
1 & 0
\end{array}\right],
$$

where $g$ is a real parameter. The corresponding $E$ has the largest eigenvalue $\lambda_{1}=\frac{1}{2}\left(\Omega+\sqrt{4+\Omega^{2}}\right)$ with $\Omega=1+g^{2}$. By comparing the spectrum of $E$ and $E^{T_{2}}$, one finds that the state is parity absent except cases of $g=0, \pm 1$. To reveal the possible symmetry and hidden string order, it is instructive to specify the four left eigenvectors of $E$ as

$$
\left|\Psi_{1,2}^{L}\right\rangle=\gamma_{1,2}|00\rangle+|11\rangle, \quad\left|\Psi_{3,4}^{L}\right\rangle=|01\rangle \pm|10\rangle,
$$

where $\gamma_{1,2}=\frac{1}{2}\left(1-g^{2} \pm \sqrt{4+\Omega^{2}}\right)$. In terms of bases (13), the transformation $S\left(k_{i}\right)=\sum_{i=1}^{4} k_{i}\left|L_{i}\right\rangle\left\langle L_{i}\right|$ is expressed as $D^{s}\left(k_{i}\right)=\left(\begin{array}{cc}B_{1} & 0 \\ 0 & B_{2}\end{array}\right)$, in which $B_{2}=\left(\begin{array}{cc}2 k_{3} & 0 \\ 0 & 2 k_{4}\end{array}\right)$ and

$$
B_{1}=\frac{1}{2} \sum_{i=1}^{2}\left[\begin{array}{cc}
k_{i}\left(1+\gamma_{i}\right)^{2} & i k_{i}\left(1-\gamma_{i}^{2}\right) \\
i k_{i}\left(\gamma_{i}^{2}-1\right) & k_{i}\left(1-\gamma_{i}\right)^{2}
\end{array}\right]
$$

By substituting it into the condition $D^{s}\left(k_{i}\right)\left[D^{s}\left(k_{i}\right)\right]^{T}=$ $I$, one derives

$$
k_{1}=\frac{\sqrt{-\gamma_{1} \gamma_{2}}}{\gamma_{1}^{2}-\gamma_{1} \gamma_{2}}, \quad k_{2}=\frac{\sqrt{-\gamma_{1} \gamma_{2}}}{\gamma_{2}^{2}-\gamma_{1} \gamma_{2}}, \quad k_{3}=k_{4}=\frac{1}{2} .
$$

Thus one can infer that the specified operator $S\left(k_{i}\right)$ falls into $\mathrm{SL}(2, \mathrm{C}) \otimes \mathrm{SL}(2, \mathrm{C})$ according to the described group Homomorphism. Simply, the decomposition $S\left(k_{i}\right)=$ $X_{1} \otimes X_{2}$ of this case can be obtained by expressing $S\left(k_{i}\right)$ in the conventional bases $\{|00\rangle,|01\rangle,|10\rangle,|11\rangle\}$ as it has a diagonal form $S\left(k_{i}\right)=\operatorname{diag}\left\{\Omega^{\frac{1}{2}}, 1,1, \Omega^{-\frac{1}{2}}\right\}$. The yielded $X_{1,2}$ and $U_{P}$ satisfying Eq. (9) turn out to be

$$
X_{1,2}=\operatorname{diag}\left\{\Omega^{\frac{1}{4}}, \Omega^{-\frac{1}{4}}\right\}, U_{P}(g)=|0\rangle\langle 0|-i e^{i \frac{\pi}{2} \mathbf{n} \cdot \sigma},
$$

where the Pauli operator $\sigma$ is defined in a two-state space $\{|+\rangle,|-\rangle\}$ and the reflection is taken in the Bloch space along $\mathbf{n}=(\cos \theta, 0,-\sin \theta)$ with $\theta=\arctan \frac{1}{g}$. As a consequence, the string order parameters $\mathrm{SO}_{G P}^{\alpha}$ of $|\Psi(g)\rangle$ are figured out in Fig. 1.

The parent Hamiltonian with $k$-local interactions always exists for a given MPS as $k>2 \log _{d} D^{9}$. It can be constructed by a sum of positive operators supported in the null space of a Hermitian matrix $\mathcal{A A}^{\dagger}$, where $\mathcal{A}$ is a $d^{k} \times D^{2}$ matrix with $\mathcal{A}_{\alpha \beta}^{i_{1} \cdots i_{k}} \equiv\left(A^{i_{1}} \cdots A^{i_{k}}\right)_{\alpha \beta}$. For the 


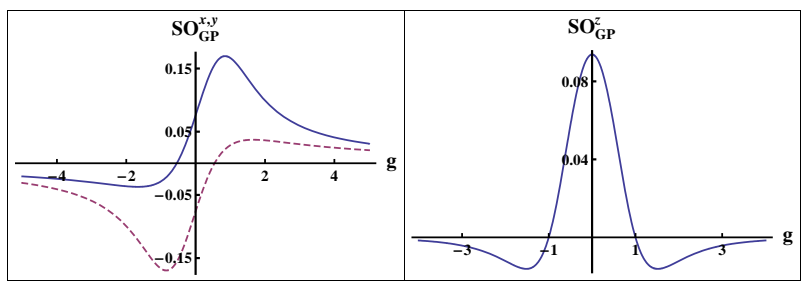

FIG. 1: The transverse string order parameters $\mathrm{SO}_{G P}^{x}$ (solid line in the left), $\mathrm{SO}_{G P}^{y}$ (dashed line in the left) and the longitudinal $\mathrm{SO}_{G P}^{z}$ (right) in the state $|\Psi(g)\rangle$ specified by Eq. (14). It is shown that there is $\mathrm{SO}_{G P}^{x}(-g)=-\mathrm{SO}_{G P}^{y}(g)$.

state $|\Psi(g)\rangle$ specified by Eq. (14) one gets a Hamiltonian with $k=2$, i.e., $H=\sum_{i} h(i, i+1)$. As $g=1$, the detailed form of $h$ is expressed as

$$
\begin{aligned}
h= & \sum_{\mu=x, y}\left[\left\{S_{i}^{\mu} S_{i+1}^{\mu}, S_{i}^{z} S_{i+1}^{z}\right\}_{+}+S_{i}^{\mu} S_{i+1}^{\mu}\left(S_{i}^{z}+S_{i+1}^{z}\right)\right] \\
& -\frac{1}{2} S_{i}^{z} S_{i+1}^{z}-\frac{1}{2}\left(A_{i} B_{i+1}+B_{i} A_{i+1}\right)+\frac{1}{2} K
\end{aligned}
$$

where $A=\left(S^{x}\right)^{2}-\left(S^{y}\right)^{2}, B=\left(S^{z}\right)^{2}-S^{z}$ and $K=$ $\left(S_{i}^{z} S_{i+1}^{z}\right)^{2}+\left(S_{i}^{+} S_{i+1}^{-}\right)^{2}+\left(S_{i}^{-} S_{i+1}^{+}\right)^{2}$. The state $|\Psi(1)\rangle$ turns to be a unique ground state of the model by construction 9,17 and it has dual symmetries under the parity and local spin reflection $U_{P}(1)$ [cf. Eq. (18)]. Consequently, the system at this point has non-vanishing string order via both passages of the parity and the local unitary, e.g., they are obtained as $\mathrm{SO}_{P}^{x}=\frac{3}{8}$, and $\mathrm{SO}_{D}^{x}=\frac{\sqrt{2}}{8}$, respectively.

Summing up, the symmetry related to spatial reflection has been investigated for quantum spin chains and a set of renewed string order was proposed. The quantity is shown applicable to characterize a variety of physical systems owing to the particular role of spatial reflection in spin lattice systems. Furthermore, revelation of the generalized parity symmetry is important in many aspects. Besides that it can be exploited to reduce computational costs in numerical algorithm, we build an explicit scheme to determine it for MPSs so that one can construct ansatz models with presumed properties. In addition, the string correlation was shown related to the concept of localizable entanglement ${ }^{20}$, a quantity reflecting the quality of a quantum channel. It would be of interest to explore further the role of the string order variants in the context of quantum information theory.

The author thanks Peng Li for helpful discussions. This work was supported by the NSFC under grant Nos. 10604043 and 10874254.
1 M. den Nijs and K. Rommelse, Phys. Rev. B 40, 4709 (1989).

2 F.D.M. Haldane, Phys. Rev. Lett. 50, 1153 (1983); Phys. Lett. 93A, 464 (1983).

3 T. Kennedy and H. Tasaki, Phys. Rev. B 45, 304 (1992); Commun. Math. Phys. 147, 431 (1992).

4 K. Hida, Phys. Rev. B 45, 2207 (1992); T. Barnes and J. Riera, Phys. Rev. B 50, 6817 (1994); E.H. Kim, G. Fath, J. Solyom, and D.J. Scalapino, Phys. Rev. B 62, 14965 (2000); F. Anfuso and A. Rosch, Phys. Rev. B 75, 144420 (2007); E. Berg, E.G. Dalla Torre, T. Giamarchi, and E. Altman, Phys. Rev. B 77, 245119 (2008); S.S. Gong and G. Su, Phys. Rev. 78, 104416 (2008).

5 I. Affleck, T. Kennedy, E.H. Lieb, and H. Tasaki, Phys. Rev. Lett. 59, 799 (1987).

6 M. Greiter, S. Rachel, and D. Schuricht, Phys. Rev. B 75 , 060401(R) (2007); D. Schuricht and S. Rachel, Phys. Rev. B 78, 014430 (2008).

7 H.H. Tu, G.M. Zhang, and T. Xiang, Phys. Rev. B 78, 094404 (2008)

8 D. Perez-Garcia, M.M. Wolf, M. Sanz, F. Verstraete, and J.I. Cirac, Phys. Rev. Lett. 100, 167202 (2008).

9 M. Fannes, B. Nachtergaele, and R.F. Werner, Europhys. Lett. 10, 633 (1989); Commun. Math. Phys. 144, 443 (1992); A. Klumper, A. Schadschneider, and J. Zittartz,
J. Phys. A 24, L955 (1991).

10 G. Vidal, Phys. Rev. Lett. 91, 147902 (2003).

11 F. Verstraete and J.I. Cirac, Phys. Rev. B 73094423 (2006); S. Iblisdir, R. Orus, and J.I. Latorre, Phys. Rev. B 75, 104305 (2007).

12 S.R. White, Phys. Rev. Lett. 69, 2863 (1992); Phys. Rev. B 48, 10345 (1993).

13 U. Schollwöck, Rev. Mod. Phys. 77, 259 (2005).

14 S. Ostlund and S. Rommer, Phys. Rev. Lett. 75, 3537 (1995); S. Rommer and S. Ostlund, Phys. Rev. B 55, 2164 (1997).

15 L.-X. Cen and Z. D. Wang, Europhys. Lett. 82, 40003 (2008)

16 D. Scalapino, S.C. Zhang, and W. Hanke, Phys. Rev. B 58, 443 (1998).

17 M.M. Wolf, G. Ortiz, F. Verstraete, and J.I. Cirac, Phys. Rev. Lett. 97, 110403 (2006).

18 L.-X. Cen, X.-Q. Li and Y.J. Yan, J. Phys. A 36, 12267 (2003).

19 C.H. Bennett, D.P. DiVincenzo, J.A. Smolin and W.K. Wootters, Phys. Rev. A 54, 3824 (1996).

20 F. Verstraete, M.A. Martin-Delgado, and J.I. Cirac, Phys. Rev. Lett. 92, 087201 (2004); L.C. Venuti and M. Roncaglia, Phys. Rev. Lett. 94, 207207 (2005). 